\title{
Measurement of deeply virtual Compton scattering (DVCS) cross sections with CLAS
}

\author{
Hyon-Suk Jo*t \\ IPN Orsay, 91406 Orsay, France \\ E-mail: jo@ipno.in2p3.fr
}

\begin{abstract}
The generalized parton distributions (GPDs) provide a new description of the complex internal structure of the nucleon in terms of its elementary constituents, the quarks and the gluons. The GPDs describe the correlation between the transverse position and the longitudinal momentum fraction of the partons in the nucleon, extending the information obtained from the measurements of the form factors and the parton distribution functions. Deeply virtual Compton scattering (DVCS), the electroproduction of a real photon from a single quark in the nucleon, $e N \rightarrow e N \gamma$, is the most straightforward exclusive process that allows access to the GPDs. A dedicated experiment to study DVCS with the CLAS detector at Jefferson Lab (JLab) has been carried out using a $5.776 \mathrm{GeV}$ polarized electron beam and an unpolarized hydrogen target, allowing us to collect DVCS events in the widest kinematic range ever explored in the valence region: $1<Q^{2}<4.6 \mathrm{GeV}^{2}, 0.1<x_{B}<0.58$, and $0.09<-t<3 \mathrm{GeV}^{2}$. We present preliminary results on the extraction of the DVCS/BH $e p \rightarrow e p \gamma$ cross sections.
\end{abstract}

Sixth International Conference on Quarks and Nuclear Physics,

April 16-20, 2012

Ecole Polytechnique, Palaiseau, Paris

\footnotetext{
* Speaker.

${ }^{\dagger}$ for the CLAS Collaboration.
} 


\section{Introduction}

Generalized parton distributions (GPDs) take the description of the complex internal structure of the nucleon to a new level by providing access to, among other things, the correlations between the transverse position and longitudinal momentum distributions of the partons in the nucleon. They also give access to the orbital momentum contribution of partons to the spin of the nucleon.

GPDs can be accessed via deeply virtual Compton scattering (DVCS) and exclusive meson electroproduction, processes where an electron interacts with a parton from the nucleon by the exchange of a virtual photon, and that parton radiates a real photon (in the case of DVCS) or hadronizes into a meson (in the case of deeply virtual meson production). The amplitude of the process can be factorized into a hard-scattering part, exactly calculable in $\mathrm{pQCD}$ or QED, and a non-perturbative part, representing the soft structure of the nucleon, parametrized by the GPDs. At leading twist and leading order approximation, there are four independent quark helicity conserving GPDs for the nucleon: $H, E, \tilde{H}$ and $\tilde{E}$. These GPDs are functions depending on three variables $x$, $\xi$ and $t$, among which only $\xi$ and $t$ are experimentally accessible. The quantities $x+\xi$ and $x-\xi$ represent respectively the longitudinal momentum fractions carried by the initial and final parton. The variable $\xi$ is linked to the Bjorken variable $x_{B}$ through the asymptotic formula: $\xi=\frac{x_{B}}{2-x_{B}}$. The variable $t$ is the squared momentum transfer between the initial and final nucleon. Since the variable $x$ is not experimentally accessible, only Compton form factors (CFFs), $\mathscr{H}, \mathscr{E}, \tilde{\mathscr{H}}$ and $\tilde{\mathscr{E}}$, whose real parts are weighted integrals of GPDs over $x$ and whose imaginary parts are combinations of GPDs at $x= \pm \xi$, can be extracted.

The reader is referred to Refs. [1-11] for detailed reviews of the GPDs and the theoretical formalism.

\section{Deeply Virtual Compton Scattering}

Among the exclusive reactions allowing access to GPDs, DVCS, which corresponds to the electroproduction of a real photon off a nucleon $e N \rightarrow e N \gamma$, is the key reaction since it offers the simplest, most straightforward theoretical interpretation in terms of GPDs.
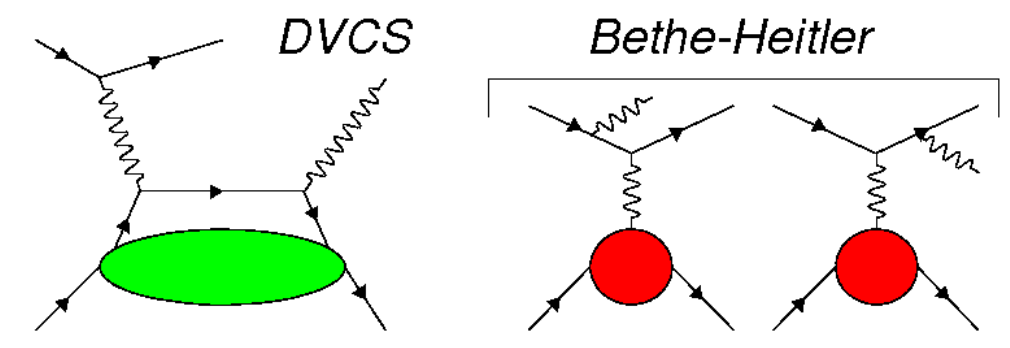

Figure 1: One of the two handbag diagrams for DVCS (left) and diagrams for Bethe-Heitler (right). These two processes contribute to the amplitude of the $e N \rightarrow e N \gamma$ reaction.

The DVCS amplitude interferes with the amplitude of the Bethe-Heitler $(\mathrm{BH})$ process which leads to the exact same final state. In the $\mathrm{BH}$ process, the real photon is emitted by either the incoming or the scattered electron while in the case of DVCS, it is emitted by the target nucleon (see 
Figure 1). Although these two processes are experimentally indistinguishable, the $\mathrm{BH}$ process is well-known and exactly calculable in QED. At current JLab energies $(6 \mathrm{GeV})$, the $\mathrm{BH}$ process is dominant in most of the phase space but the DVCS process can be accessed via the interference term arising from the two processes. With a polarized beam or/and a polarized target, different types of asymmetries can be extracted: beam-spin asymmetry $\left(A_{L U}\right)$, longitudinally polarized target-spin asymmetry $\left(A_{U L}\right)$, transversely polarized target-spin asymmetry $\left(A_{U T}\right)$, and double-spin asymmetries $\left(A_{L L}, A_{L T}\right)$. Each type of asymmetry gives access to a different combination of CFFs, being mostly sensitive to one or two particular CFF(s). Single-spin asymmetries give access to the imaginary part of the CFFs and double-spin asymmetries to their real part. The DVCS/BH $e N \rightarrow e N \gamma$ unpolarized cross section is sensitive to both the real part and the imaginary part of the CFFs. The polarized cross section difference is linearly proportional to the imaginary part of the CFFs.

\section{The e1-DVCS experiment}

The first DVCS results published by the CLAS collaboration were extracted using data from non-dedicated experiments: beam-spin asymmetries in 2001 [12] and longitudinally polarized target-spin asymmetries in 2006 [13]. In 2005, the first part of the e1-DVCS experiment was carried out in Hall B at JLab using the CLAS spectrometer [14] and an additional electromagnetic calorimeter, specially designed and built for the experiment, and made of 424 lead-tungstate scintillating crystals read out via avalanche photodiodes (see Figure 2). This additional calorimeter was located at forward angles, not covered by CLAS, where the DVCS/BH photons are mostly emitted. This first CLAS experiment dedicated to DVCS was fully exclusive and ran using a $5.766 \mathrm{GeV}$ polarized electron beam and a liquid-hydrogen target.

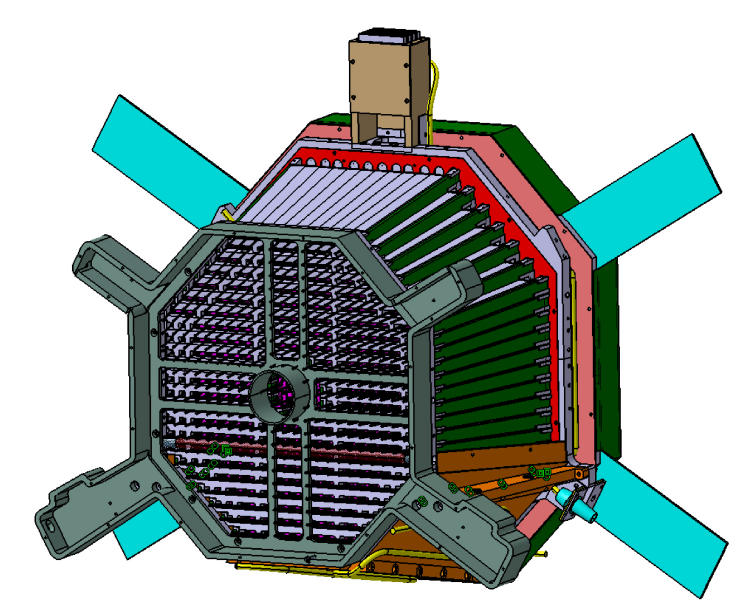

Figure 2: The DVCS electromagnetic calorimeter, specially designed and built for the e1-DVCS experiment to detect DVCS/BH photons emitted at forward angles. It was later used in two other experiments dedicated to DVCS.

From the e1-DVCS data, CLAS published in 2008 the largest set of DVCS beam-spin asymmetries ever extracted in the valence region [15]. This report presents preliminary results on the extraction of DVCS/BH $e p \rightarrow e p \gamma$ cross sections using the e1-DVCS data [16]. 


\section{Extraction of DVCS/BH $e p \rightarrow e p \gamma$ cross sections}

The e1-DVCS data covers the range: $1<Q^{2}<4.6 \mathrm{GeV}^{2}, 0.1<x_{B}<0.58$, and $0.09<-t<$ $3 \mathrm{GeV}^{2}$. We have analyzed the $e p \rightarrow e p \gamma$ reaction and are in the process of extracting 4-fold differential cross sections $\frac{d^{4} \sigma}{d Q^{2} d x_{B} d t d \Phi}$ in bins in $\left(Q^{2}, x_{B},-t, \Phi\right)$. Figure 3 shows the kinematic coverage of the e1-DVCS data. On the left, is shown the phase space $Q^{2}$ as a function of $x_{B}$, the lines representing the 13 bins in the $\left(Q^{2}, x_{B}\right)$ plane chosen for this analysis. Although we extracted the DVCS/BH ep $\rightarrow e p \gamma$ cross sections for all bins, we show preliminary results only for three representative bins (highlighted in red on the figure): Bin 2, at low $Q^{2}$ and $x_{B}$, Bin 5, at intermediate $Q^{2}$ and $x_{B}$, and Bin 10, at relatively high $Q^{2}$ and $x_{B}$. On the right, is shown $-t$ as a function of $x_{B}$, the horizontal lines representing the 12 bins in $-t$.
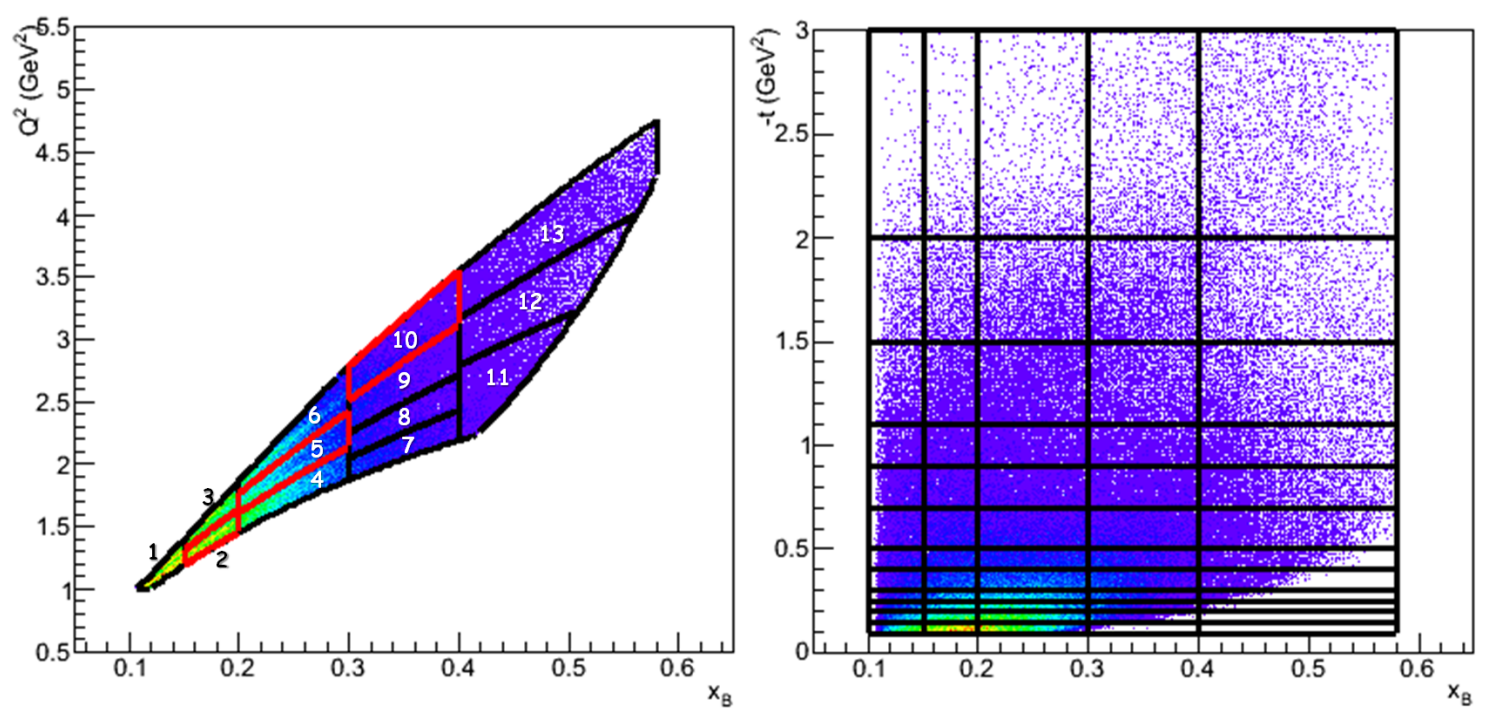

Figure 3: Kinematic coverage of the e1-DVCS data. On the left: $Q^{2}$ as a function of $x_{B}$, showing the 13 bins in the $\left(Q^{2}, x_{B}\right)$ plane chosen for the analysis. The bins highlighted in red (Bin 2, Bin 5 and Bin 10) are the ones for which results are shown in this report. On the right: $-t$ as a function of $x_{B}$, with the horizontal lines representing the 12 bins in $-t$.

We present preliminary results for the DVCS/BH $e p \rightarrow e p \gamma$ unpolarized cross sections as a function of $\Phi$ (the angle between the lepton-scattering plane and the hadronic plane) for Bin 2, Bin 5 and Bin 10 and three bins in $-t$ (see Figure 4). The results are shown with 24 bins in $\Phi$ although the analysis was performed using 72 bins that were later merged. Only the statistical errors are shown since systematic studies are still underway. The curves represent the $\mathrm{BH}$ calculation integrated over each 4-dimensional bin. The difference between the curves and the data can be attributed to the DVCS process and the interference term, which are therefore a significant contribution. The polarized cross section difference was extracted as well. Figure 5 shows preliminary results for Bin 5 and three bins in $-t$. 

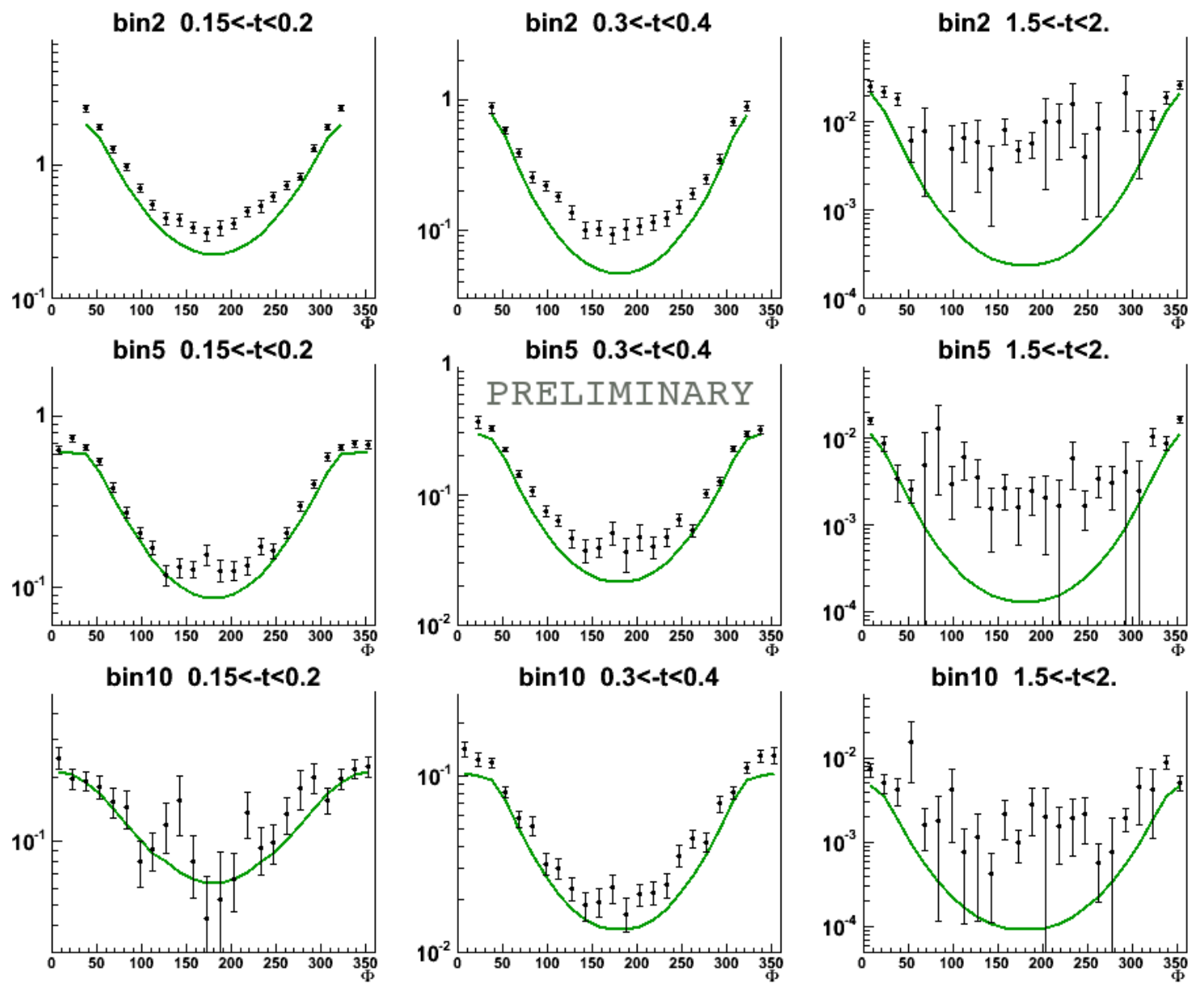

Figure 4: Preliminary results for the $e p \rightarrow e p \gamma$ unpolarized cross sections $\left(\mathrm{in} n \mathrm{nb} / \mathrm{GeV}^{4}\right.$ ) as a function of $\Phi$, for Bin 2, Bin 5 and Bin 10 and three bins in $-t$. The curves represent the BH calculation, integrated over each 4-dimensional bin.
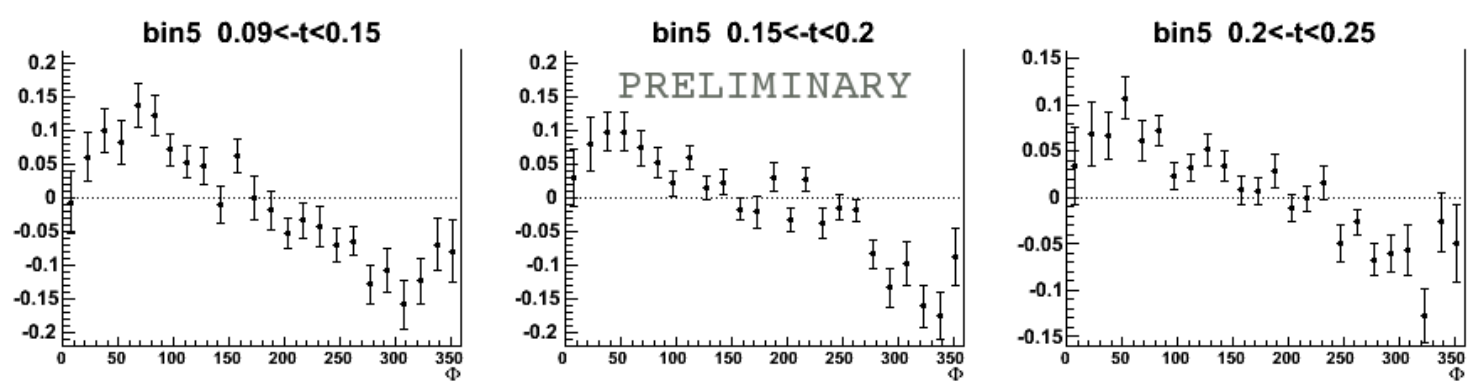

Figure 5: Preliminary results for the $e p \rightarrow e p \gamma$ polarized cross section differences (in $\mathrm{nb} / \mathrm{GeV}^{4}$ ) as a function of $\Phi$, for Bin 5 and three bins in $-t$. 


\section{Summary}

DVCS/BH ep $\rightarrow e p \gamma$ unpolarized and polarized cross sections were extracted in the widest kinematic range ever explored in the valence region. Preliminary results for the unpolarized cross sections are compared to the corresponding BH calculations. Preliminary results for the polarized cross section differences are shown as well. These new results will allow the extraction of CFFs, using global fitting codes, and will thus provide constraints on GPD models over a very large kinematic range.

\section{Acknowledgments}

Thanks to the organizing committee of the QNP2012 conference and to the conveners of the parallel session for the opportunity to give this presentation.

\section{References}

[1] D. Müller, D. Robaschik, B. Geyer, F.-M. Dittes, and J. Horejsi, Fortschr. Phys. 42, 101 (1994).

[2] X. Ji, Phys. Rev. Lett. 78, 610 (1997); Phys. Rev. D 55, 7114 (1997).

[3] A.V. Radyushkin, Phys. Lett. B 380 (1996) 417; Phys. Rev. D 56, 5524 (1997).

[4] J.C. Collins, L. Frankfurt and M. Strikman, Phys. Rev. D 56, 2982 (1997).

[5] K. Goeke, M.V. Polyakov and M. Vanderhaeghen, Prog. Part. Nucl. Phys. 47, 401 (2001).

[6] M. Diehl, Phys. Rept. 388, 41 (2003).

[7] A.V. Belitsky, A.V. Radyushkin, Phys. Rept. 418, 1 (2005).

[8] M. Guidal, Prog. Part. Nucl. Phys. 61, 89 (2008).

[9] M. Vanderhaeghen, P.A.M. Guichon, and M. Guidal, Phys. Rev. D 60, 094017 (1999).

[10] M. Guidal, M.V. Polyakov, A.V. Radyushkin and M. Vanderhaeghen, Phys. Rev. D 72, 054013 (2005).

[11] A. Belitsky, D. Müller and A. Kirchner, Nucl. Phys. B 629, 323 (2002).

[12] S. Stepanyan et al (CLAS Collaboration), Phys. Rev. Lett. 87, 182002 (2001).

[13] S. Chen et al. (CLAS Collaboration), Phys. Rev. Lett. 97, 072002 (2006).

[14] B. Mecking et al., Nucl. Instrum. Meth. A 503, 513 (2003).

[15] F.X. Girod et al (CLAS Collaboration), Phys. Rev. Lett. 100, 162002 (2008).

[16] H.S. Jo, Ph.D. thesis, Université Paris-Sud, Orsay, France (2007). 